\title{
Emission Properties Related to Distinct Phases of Sol-Gel Dip-Coating Titanium Dioxide, and Carrier Photo-Excitation in Different Energy Ranges
}

\author{
Roberto de Aguiar Ramos Jr ${ }^{a}$, Miguel Henrique Boratto ${ }^{a}$, Maximo Siu Lit, Luis Vicente de Andrade Scalvit * \\ ${ }^{a}$ Physics Department and POSMAT, São Paulo State University - UNESP, Bauru, SP, Brazil \\ ${ }^{b}$ Institute of Physics of São Carlos, University of São Paulo - USP, São Carlos, SP, Brazil
}

Received: September 16, 2016; Revised: December 19, 2016; Accepted: February 12, 2017

\begin{abstract}
Titanium dioxide $\left(\mathrm{TiO}_{2}\right)$ in the form of pellets (pressed powder) and thin films are investigated, revealing the presence of distinct phases: mainly anatase and rutile. Characterization of optical, structural and electrical properties were carried out on samples submitted to different sort of thermal annealing (TA), at distinct temperatures, 500 and $1000^{\circ} \mathrm{C}$, due to their influence on the obtained phases. TA temperature along with pressure application for sample conformation, determine the bands present in the photoluminescence (PL) spectra, being about $550 \mathrm{~nm}$, characteristic of anatase phase and $800 \mathrm{~nm}$, related to the presence of rutile phase. The bandgap of thin films is determined from optical absorbance data, yielding $3.4 \mathrm{eV}$ for anatase phase (indirect transition), and $2.9 \mathrm{eV}$ for rutile phase (direct transition). Besides, irradiation with monochromatic light strongly affects the thin film conductivity, but the energy range (above or below the bandgap energy) does not seem to affect the behavior, which is associated with the excitation of intrabandgap states or crystallites belonging to phases with distinct bandgaps.
\end{abstract}

Keywords: titanium dioxide, photoluminescence, phase transition, conductivity decay

\section{Introduction}

Titanium dioxide $\left(\mathrm{TiO}_{2}\right)$ is an oxide semiconductor material with wide bandgap of the order of $3.3 \mathrm{eV}^{1,2}$. The bandgap may have direct or indirect electronic transition ${ }^{1,3}$ depending on the crystal structure: anatase, rutile and brookite ${ }^{4}$. Its n-type semiconducting nature comes from the non stoichiometric $\mathrm{TiO}_{2-\mathrm{x}}$ composition, which originates oxygen vacancies $(\mathrm{x} \cong$ 0.01 ), leading the $\mathrm{Ti}^{3+}$ ions to behave as a donor impurity ${ }^{5}$. This material is largely investigated due to a wide range of applications, such as solar cells, water purification ${ }^{6}$, or gas sensor $^{7}$, etc ${ }^{8}$. High refraction index of the anatase phase allows its use as a thin film reflective coating on Si solar cells $\mathrm{s}^{4}$. The rutile phase has a lower Gibbs free energy in the bulk than the anatase phase, however, the surface Gibbs free energy of the rutile phase is higher than the anatase phase, therefore, $\mathrm{TiO}_{2}$ begins its nucleation in the anatase phase ${ }^{9}$ at low temperatures, range where it presents higher stability. However, the rutile phase is more stable but initiates its nucleation at higher temperatures ${ }^{10}$. The phase transition depends on several factors such as particle size, sol-gel pH, surface energy, synthetic route ${ }^{11}$, and doping impurities ${ }^{2}$. A simple method for obtaining the rutile phase is through the heat treatment of the anatase phase, for the reason that the thermal energy facilitates the rearrangement of atoms ${ }^{2}$, leading to the phase transition from $400^{\circ} \mathrm{C}$ to $1200^{\circ} \mathrm{C}^{2,12,13}$.

$\mathrm{TiO}_{2}$ can be synthesized by several different methods, including liquid phase processing techniques, which have

*e-mail: scalvi@fc.unesp.br the advantage of stoichiometry control, in order to produce homogeneous materials. Among these methods, the solgel process has been widely used because it provides an appropriate level of purity and high flexibility for doping introduction ${ }^{4}$. There are two sorts of known sol-gel method: the alkoxide route and non-alkoxide route. The non-alkoxide route uses inorganic salts (such as nitrates, chlorides, acetates, carbonates, acetylacetonates, etc. $)^{4,14}$, which requires an additional removal of the inorganic anion, whereas the alkoxide route (the most employed) uses metal alkoxides as precursor reagents ${ }^{4,15}$. In general, this latter method involves the formation of sol or gel or precipitates by hydrolysis and condensation of titanium alkoxides ${ }^{4}$. $\mathrm{TiO}_{2}$ thin films also are frequently obtained by chemical vapor deposition (CVD) ${ }^{16}$ and spray pyrolysis deposition (SPD) $)^{17}$. More recently, $\mathrm{TiO}_{2}$ have been produced in the form of nanotubes ${ }^{18}$, and there have been reports on the growth of nanotube films ordered by anodization ${ }^{19}$, because with this highly ordered arrangement the electron mobility can be improved ${ }^{20}$.

Electrical properties such as resistivity, are changed considerably due to the thermal oxidation or reduction of the films, which shows that the concentration of charge carriers in the thin film is dependent on non-stoichiometry ${ }^{21}$. Optical properties such as photoluminescence (PL) and the nature of the electronic transition depend on the atomic structure. For better implementation and use of the optical and electrical properties of this material in devices, the determination of the emission bands characteristic of each phase, and its identification as well as the stoichiometry properties are 
fundamental. The PL of the anatase phase is related to two main bands: emission from self trapped excitons (440-510 $\mathrm{nm}$ ) and deep levels formed by oxygen vacancies (515-560 $\mathrm{nm})^{22}$. The self trapped excitons decrease the total energy of excitons through lattice relaxation, leading to emission energy lower than the bandgap ${ }^{21}$. On the other hand, the PL emission of the rutile structure comes from deep levels related to the excited states of $\mathrm{Ti}^{3+}(800-880 \mathrm{~nm})^{23}$.

In this paper, we report results of X-ray diffraction (XRD), Thermogravimetry / Differential Thermal Analysis (TG / DTA), Photoluminescence (PL) and decay of photoinduced electrical current, which allowed identifying the emission bands characteristic of each phase and the transition anatase/rutile. Properties of photo-induced conductivity, related to the time response of titanium dioxide thin films for excitation with different wavelengths are also obtained. This material exhibits a high sensitivity when excited, and a fast decay to the ground state when the excitation is removed, showing suitable properties for applications in opto-electronic speedy devices.

\section{Experimental}

The titanium dioxide solution was obtained through reactions of hydrolysis and condensation of titanium isopropoxide alkoxide (IV) using a high molar ratio of water:alkoxide (200:1), isopropanol as co-solvent, $\mathrm{HNO}_{3}$ as a catalyst and Triton X-100 as surfactant. This solution allows obtaining $\mathrm{TiO}_{2}$ thin films by dip-coating technique on soda-lime glass substrates, with immersion/emersion rate of $10 \mathrm{~cm} / \mathrm{min}$. Four $\mathrm{TiO}_{2}$ layers were deposited, being thermally heated after each deposited layer at $80^{\circ} \mathrm{C}$ for 10 minutes. A final thermal annealing is provided at $500^{\circ} \mathrm{C}$ for 2 hours. Thin films were also deposited on quartz, but in this case the final thermal annealing was at $1000^{\circ} \mathrm{C}$. The powders were obtained from heating the solution at $85^{\circ} \mathrm{C}$ for 2 hours in order to provide evaporation of the whole dispersant. Evaporation leads to formation of tiny crystals, which were mashed, generating the $\mathrm{TiO}_{2}$ powder. These powders were compressed with $7 \times 10^{3} \mathrm{Kg} / \mathrm{cm}^{2}$ to form pellets, being submitted to a distinct sequence of thermal annealing to originate 4 distinct samples. Table 1 shows the order and temperature of thermal annealing of these samples.

Analysis of X-ray Diffraction (XRD) was performed on a Rigaku diffractometer (Model D / Max - 2100 / PC) operating with $\mathrm{Cu} \mathrm{K} \mathrm{K}_{\alpha}$ radiation $(1.5405 \AA$ ) and $\mathrm{Ni}$ filter for reducing unwanted $\mathrm{K}_{\beta}$ radiation. The measurement range was from 20 to $80^{\circ}$ with a scanning rate of $2 \% \mathrm{~min}$, in the $2 \theta$ mode for films (fixed incident angle of $1.5^{\circ}$ ). Data were analyzed with the aid of the Match software (Crystal Impact), using PDF files \#01-089-4921 and \#01-089-4920 for anatase and rutile phases, respectively. The average crystallite size was estimated using the Scherrer equation ${ }^{24}$.Transmittance and absorbance were performed in a Perkin
Table 1. Thermal annealing of the samples, before and after pellet compression (PC), as function of Temperature (T) and time (t).

\begin{tabular}{lcc}
\hline Sample & $\begin{array}{c}\text { TA after PC: } \\
\mathrm{T}\left({ }^{\circ} \mathrm{C}\right) / \mathrm{t}(\mathrm{h})\end{array}$ & $\begin{array}{c}\text { TA before PC: } \\
\mathrm{T}\left({ }^{\circ} \mathrm{C}\right) / \mathrm{t}(\mathrm{h})\end{array}$ \\
\hline P1 & --- & $1000 / 5$ \\
P2 & --- & $500 / 5$ \\
P3 & $500 / 5$ & $500 / 2$ \\
P4 & $1000 / 5$ & $1000 / 1$ \\
\hline
\end{tabular}

Elmer spectrophotometer (Lambda Model 1050 UV / VIS / NIR) in the range of 200 to $3300 \mathrm{~nm}$. Evaluation of the optical bandgap was performed by using Tauc method ${ }^{25}$. For photoluminescence (PL) measurements, the line 350.7 $\mathrm{nm}$ of a $\mathrm{Kr}^{+}$laser was used (Innova model 200), with output power of about $500 \mathrm{~mW}$, being the power that reaches the sample of about $14 \mathrm{~mW}$, after the beam travel through several optical components (a prism, four mirrors, two lenses, $138 \mathrm{~Hz}$ chopper and iris). The data acquisition is performed using a PC interfaced to control the monochromator step motor (Thermo Jarrell-Ash) and receives signal from a lock-in (SR530 model 16 Stanford Research Systems).

To perform electrical measurements on the thin films, Indium (In) electrodes were deposited at low pressure $\left(10^{-5}\right.$ mbarr) in a BOC Edwards evaporation system (model 500 Auto system). Current decay measurements were performed in a Janis closed-He cryostat coupled to a He compressor (HC-2 model, Intermagnetic General Corp. - ODA) ${ }^{26}$, and a temperature controller (model 330, Lake Shore Cryotronics). Optical excitation of samples were provided with distinct light sources: deuterium lamp coupled to an interference filter with peak at $270 \mathrm{~nm}$ (above $\mathrm{TiO}_{2}$ bandgap energy), and also InGaN LED with wavelength in the range 440-460nm, and central peak of $450 \mathrm{~nm}$ (energy below bandgap of $\mathrm{TiO}_{2}$ anatase phase). Analysis of TG / DTA(STA 409 cell, Netzsch) was carried out for $\mathrm{TiO}_{2}$ powder from 40 to $1000^{\circ} \mathrm{C}$ with rate of $10^{\circ} \mathrm{C} / \mathrm{min}$ in a controlled $\mathrm{N}_{2}$ atmosphere.

\section{Results and discussion}

It must be recalled that Table 1 , described in the experimental section, defines the history of each pellet sample. The pellets P1 and P2 were thermally annealed (TA) only after pressing, whereas pellets $\mathrm{P} 3$ and $\mathrm{P} 4$ were annealed before and after applying pressure. Figure 1 shows X-rays diffractograms for these pellet samples. The diffraction peaks related to $\mathrm{P} 1$ and $\mathrm{P} 4$ samples with TA at $1000^{\circ} \mathrm{C}$, refer to the rutile phase of $\mathrm{TiO}_{2}$ with superior intensity on the (110) plane. Sample P1, predominantly rutile phase, still presents a less intense peak related to (200) plane of anatase phase. Samples P2 and P3, thermally annealed at $500^{\circ} \mathrm{C}$, present structure related to the anatase phase, with greater intensity in the (101) plane, but with a lower intensity peak concerning the (002) and (301) planes of rutile phase. Both crystal 


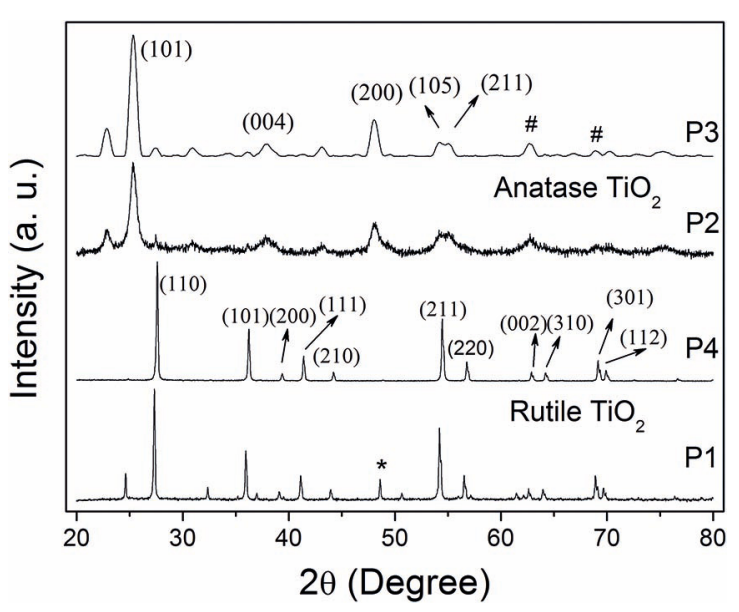

Figure 1. XRD of $\mathrm{TiO}_{2}$ pellets. The main diffraction peaks of samples $\mathrm{P} 1$ to $\mathrm{P} 4$ are displayed. Samples $\mathrm{P} 1$ and $\mathrm{P} 4$ have rutile phase (catalog JCPDS-ICDD 2003 file number 89-4920) after TT $1000^{\circ} \mathrm{C}$, but some anatase peaks (*) still appear in the P1 diffractogram. Samples P2 and P3 have the anatase phase (catalog JCPDS-ICDD 2003 file number $78-2486$ ), after TT $500^{\circ} \mathrm{C}$, but also show peaks of rutile phase (\#) .

structures presented in these samples show an incomplete phase transition, which is supposed to take place between $400^{\circ} \mathrm{C}$ and $1200^{\circ} \mathrm{C}^{13,22}$, involving TA temperatures used for all samples. This presence of two phases may be generated also by encapsulating of a structure over the other ${ }^{2}$, such as the rutile formation on anatase particles ${ }^{27}$ or rutile phase formation in the bulk of anatase phase grains ${ }^{28}$. The average crystallite size, calculated based on the 5 more intense peaks of the diffractograms of the samples, showed a significant increase from $5.1 \mathrm{~nm}$ for P2 to $9.4 \mathrm{~nm}$ for P3 sample. This crystallite size is higher for sample with two TA compared to samples with only one TA at the same temperature (after pressing). P2 and P3 samples, which were treated at the same temperature $\left(500^{\circ} \mathrm{C}\right)$, present a significant increase in the crystallite size when the sample gets a second thermal annealing. The same behavior holds for samples P1 and P4 (TA $1000^{\circ} \mathrm{C}$ ) with $38.5 \mathrm{~nm}$ of crystallite size for sample $\mathrm{P} 1$ and $39.9 \mathrm{~nm}$ for sample P4. In both cases the samples with two TA are submitted to a longer time of annealing, leading to this expected increase in the crystallite size. It is also interesting to notice the large increase in the crystallite size when the dominant phase changes from anatase to rutile. It can be clearly observed in the diffractograms (fig. 1) by the shape of the main peaks, which are rather larger for anatase-dominant samples.

For further investigation of the crystallization processes and phase transition temperatures a thermogravimetry/ differential thermal analysis (TG/DTA) measurement was carried out on the P4 sample, before any thermal annealing or pressing. This result is shown in Figure 2. There is an abrupt weight loss in the TG data, which occurs between 50 and $150^{\circ} \mathrm{C}$, associated with the endothermic peak centered

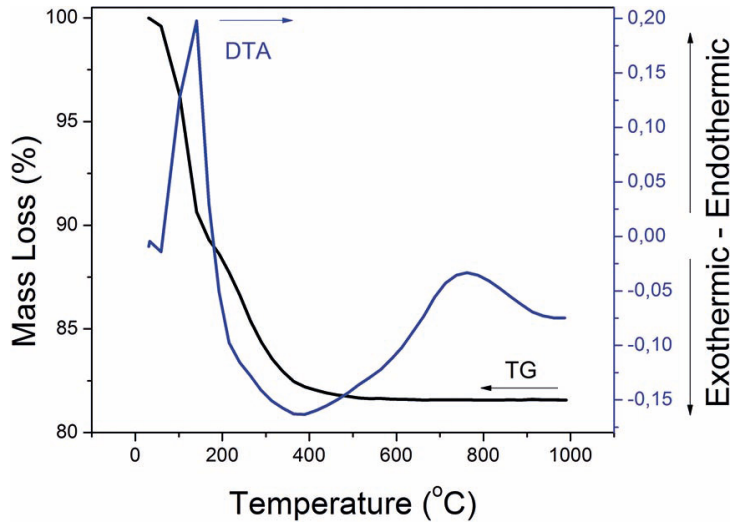

Figure 2. TG and DTA for Titanium dioxide powder previous to any thermal annealing. This powder became $\mathrm{P} 4$ sample after complete annealing as described in Table 1. The endothermic peak shown in the DTA, from $400^{\circ} \mathrm{C}$ to $1000^{\circ} \mathrm{C}$ is not accompanied by mass loss in TG, which characterizes the phase transition anatase / rutile.

at $150^{\circ} \mathrm{C}$ in DTA curve. This weight loss can be related with water desorption of the powder and solvent evaporation from the precursor solution ${ }^{29}$. The weight loss between $150^{\circ} \mathrm{C}$ and $400^{\circ} \mathrm{C}$, accompanied by an exothermic peak centered at $380^{\circ} \mathrm{C}$ in the DTA curve, may be due to transformation of peroxides into oxides, or by the evaporation of organic parts from the sample, coming from the Triton X-100, as well as solvents ${ }^{22,29}$. From $400^{\circ} \mathrm{C}$ no mass loss is recorded in $\mathrm{TG}$, but there is the appearance of an endothermic region that starts at $650^{\circ} \mathrm{C}$ and goes until $800^{\circ} \mathrm{C}$ in DTA, being centered at $750^{\circ} \mathrm{C}$, which shall represent the phase transition anatase / rutile, characterized by a free mass loss process. This phase transition anatase / rutile is confirmed by the XRD diffractograms shown in Figure 1, which shows rutile dominant peaks for samples submitted to TA temperature of $1000^{\circ} \mathrm{C}$, in good agreement with literature ${ }^{2,13,30}$.

Figure 3 shows photoluminescence (PL) data for measurement performed on $\mathrm{TiO}_{2}$ pellets with different TA. The data are in very good agreement with diffraction peaks found in all samples related to incomplete phase transition, shown in Figure 1. The observed band at $550 \mathrm{~nm}$ for $\mathrm{P} 2$ and $\mathrm{P} 3$ samples is probably related to the deepest levels of oxygen vacancies of the anatase phase ${ }^{22}$ and the band found around $800 \mathrm{~nm}$ is related to rutile phase of $\mathrm{TiO}_{2}{ }^{23,31}$. Concerning the samples treated at $500^{\circ} \mathrm{C}$, it is observed a higher intensity at $550 \mathrm{~nm}$ for P2 sample, which was thermally annealed only once, while $\mathrm{P} 3$, which has been treated two hours more than P2 has a higher relative intensity of the band at $800 \mathrm{~nm}$, which can be associated with a more developed transition of anatase to rutile phase, and also related to a decrease in oxygen vacancy concentration ${ }^{22}$. This more developed transition is also observed in the XRD data shown in figure 1. The appearance of the rutile phase even for annealing at $500^{\circ} \mathrm{C}$, although this temperature is in the beginning of transition range of anatase to rutile, it may also be related to 


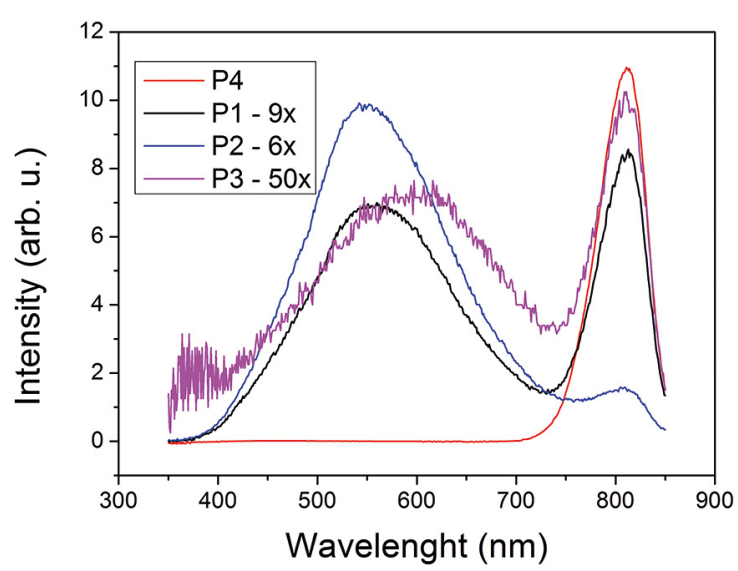

Figure 3. Photoluminescence of pellets $\mathrm{P} 1$ to $\mathrm{P} 4$. The curves are normalized with respect to intensity. Bands centered at 550 and $800 \mathrm{~nm}$ refer to anatase and rutile structures, respectively. Anatase structure is found in three samples with two phases present. Sample $\mathrm{P} 4$, with two thermal annealing (before and after pressing) presents only rutile structure.

the pressing to obtain pellets, because the pressure applied on the particles may promote the rearrangement of atoms in the crystal lattice, accelerating the phase transition. Although typically anatase / rutile transition is studied in terms of time and temperature ${ }^{2}$, the activation of anatase-rutile conversion can also be initiated by pressure increase ${ }^{4}$. On the other hand, samples treated at $1000^{\circ} \mathrm{C}(\mathrm{P} 1$ and $\mathrm{P} 4)$ present dominant rutile structure, also verified by XRD data, shown in Figure 1. These samples show the band in the $800 \mathrm{~nm}$ on the PL spectra. In the P4 sample, which had TA before and after pressing, only the rutile band is observed, which means that the second TA favored the complete phase transition. However in the P1 sample, an incomplete transition is observed in the PL spectra, with comparable intensity of the anatase and rutile bands. Again, confirmed with diffractograms shown in Figure 1, which also reveals the presence of anatase structure. Samples such as P1 and P4, formed mainly by rutile phase have larger crystallites, which are larger for this phase, as confirmed by the XRD peak width shown in figure 1. The same behavior is observed for the sample P3, which features larger crystallites than P2, due to the higher proportion of rutile phase in its structure ${ }^{2}$. It is interesting to notice that bands centered about $550 \mathrm{~nm}$ are wider than the band centered at $800 \mathrm{~nm}$. Plugaru and coworkers ${ }^{32}$ have investigated policrystalline $\mathrm{TiO}_{2}$ thermally treated in the range of 1100 to $1500^{\circ} \mathrm{C}$, under distinct atmospheres, and found results of cathode-luminescence very similar to our PL data. In that case, the transitions in the range 820 and $850 \mathrm{~nm}$ are associated with the formation of $\mathrm{Ti}^{3+}$ interstitial ions in rutile structure, and with its associated defect states, during the sintering process. On the other hand, the band about 550nm has been reported in the literature either for anatase single crystals as well as nanostructures, and has been commonly attributed to the recombination of self-trapped excitons for bandgap energy excitation, which takes place due to a strong exciton-phonon interaction. Besides, in some anatase nanostructures, the room temperature PL band was attributed to oxygen vacancies ${ }^{33}$. However, the most striking found result is that the large anatase band about $550 \mathrm{~nm}$ was found even for single crystals ${ }^{34}$, being attributed to the recombination of self-trapped excitons. Then, it seems fair to relate the observed bands in our case to intrinsic defects such as oxygen vacancies and interstitial $\mathrm{Ti}^{3+}$ ions, and exciton recombination. However, the band width may probably be related to crystallite size, as already discussed, since the narrower band takes place for the sample with larger crystallites, which means that the neighborhood in the case of smaller crystallites are more random, due to grain boundary depletion layer, which affects the defects energy distribution, leading to a larger PL band, in the case of anatase. It is also in good agreement with the XRD diffratograms, because when the anatase is the dominant phase the peak width is larger (smaller crystallites) whereas for dominant rutile phase the XRD peaks are much more well defined (much larger crystallites).

$\mathrm{TiO}_{2}$ thin films were deposited on soda-lime glass substrate and thermally annealed at $500^{\circ} \mathrm{C}$, similar to the treatment carried out for pellets, in order to investigate the anatase phase, and other thin film was deposited on quartz, and thermally annealed at $1000^{\circ} \mathrm{C}$, to investigate rutile phase, also similar to thermal treatment carried out for pellets. Structural data of XRD for films are shown in figure 4 and reveals anatase and rutile phase for films deposited on glass and quartz, respectively. Similar to the results shown in figure 1 , for pellets, the thermal annealing temperature determines the dominant phase present in the film. The crystallite size for the anatase phase is $2.5 \mathrm{~nm}$ for films deposited on glass substrates (anatase phase) and $11.7 \mathrm{~nm}$ for films deposited on quartz (rutile phase). Both crystallites are smaller than powder's, probably due to intermediate TA at $80^{\circ} \mathrm{C}$, between

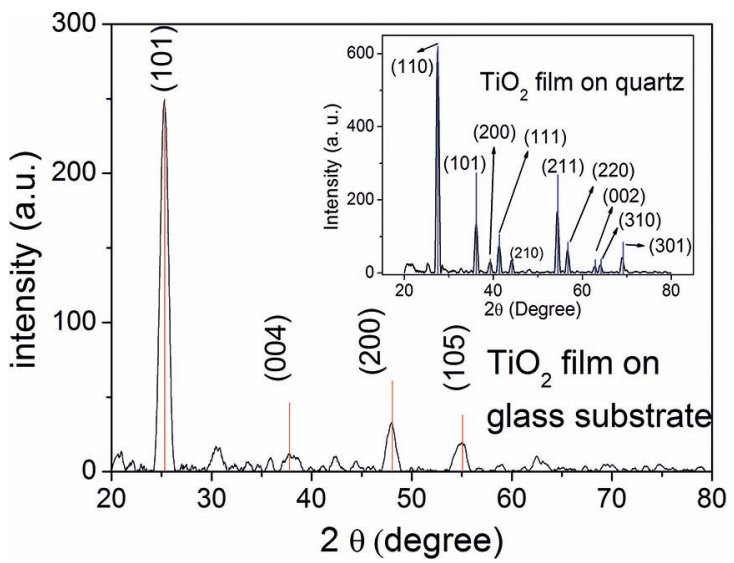

Figure 4. $\mathrm{XRD}$ of $\mathrm{TiO}_{2}$ thin film deposited on glass substrates. Red lines refer to Anatase (catalog JCPDS-ICDD 2003 file number 782486). Inset: XRD diffractogram for $\mathrm{TiO}_{2}$ film deposited on quartz substrate. Blue lines refers to rutile phase (catalog JCPDS-ICDD 2003 file number $89-4920$ ) 
each of the four film layers. It facilitates nucleation of small crystallites which do not increase with the final TA.

Figure 5 shows transmittance data and the evaluation of the indirect bandgap ${ }^{2}$ for the anatase phase film (fig. 5(a)), and the same sort of results for the rutile phase film (fig. 5(b)), in this case the bandgap is direct. The bandgap is about $3.4 \mathrm{eV}$ for anatase phase film, and the high transmittance in the UV-Vis, allowed calculation of the film thickness from the oscillations of the interference fringes ${ }^{35}$, of about 300 $\mathrm{nm}$. The rutile phase film, with direct transition, present bandgap of about $2.9 \mathrm{eV}$, in fair agreement with literature ${ }^{4}$.

Figure 6 shows the photo-induced current decay for a $\mathrm{TiO}_{2}$ thin film deposited on soda-lime glass substrate, which is held in a pressure of $10^{-3} \mathrm{mbarr}$, after being excited by irradiation with a deuterium lamp (UV), during $5 \mathrm{~min}$, through interference filter with peak at $270 \mathrm{~nm}(4.58 \mathrm{eV})$. The sample layout and the electrical measurement setup are shown in the inset of fig. 6 . In this measurement, after the light excitation removal, the sample is taken to dark conditions, when the current is measured, under an applied voltage of $20 \mathrm{~V}$. The same sort of measurement, where excitation is done with a InGaN LED is also provided, where the light source device has an average wavelength of $450 \mathrm{~nm}(2.75 \mathrm{eV})$. Both measurements show excitation to similar current magnitudes, with a large increase in conductivity due to exposure to light. Besides, in both cases there is a quick return to steady state, with percentage decay of $98 \%$ and $99 \%$ to excitations with wavelengths of 270 and $450 \mathrm{~nm}$, respectively, 20 min after removing the excitation. Surprisingly, either the excitation as well the decay show similar results, which means that two distinct sort of phenomena may be taking place: 1) excitation of intrabangap states, dominating over the electron-hole pairs, 2) the presence of particles belonging to different phases in the material, which have distinct bandgaps, and are more efficiently excited by distinct energy ranges. It is in good agreement with figure 3, which shows PL bands characteristics of distinct phases in the same sample, and with figure 5, which show distinct bandgaps for each phase, anatase $(3.4 \mathrm{eV})$ and rutile $(2.9 \mathrm{eV})$. Electrical resistivity was evaluated for this film, before and after the excitation, from resistance data and using the following configuration: $\mathrm{L}=$ $25 \mathrm{~mm}, \mathrm{~d}=5 \mathrm{~mm}$, and $\tau=300 \mathrm{~nm}$ (these dimensions are also shown in the inset of figure 6). Before the excitation, in the dark, the sample electrical conductivity was $8.3 \times 10^{-4}$ $(\Omega . \mathrm{m})^{-1}$, and after excitation for $5 \mathrm{~min}$ with the light of wavelength 270 and $450 \mathrm{~nm}$ became $5.3 \times 10^{-2}$ and $4 \times 10^{-2}$ $(\Omega . \mathrm{m})^{-1}$, respectively, an increase of the conductivity of up to 64 times due to sample illumination.

The conductivity decay as function of time shown in figure 6 can be modeled for both sort of structures (anatase and rutile). The monochromatic light source is either below bandgap energy or above, considering that the anatase phase is supposed to be dominant in this thin film sample (deposited on soda-lime glass substrate and thermally annealed at $500^{\circ} \mathrm{C}$ ). Surprisingly the same sort or excitation and decay are recorded, assuring that the generation and recombination are either more than only electron-hole pairs, coming also from intrabandgap states with lower ionization energy, or that there are crystallites of phases with bandgap about $2.75 \mathrm{eV}$ $(450 \mathrm{~nm})$, in good agreement with results of XRD diffraction as well as PL data, which shows that samples are actually a combination of crystallites belonging to both phases.

In the modeling of this sort of experiment, it is fundamental to provide temperature dependent data. It has been carried out for $\mathrm{SnO}_{2}$ thin films with several sort of doping such as Er-doped and Eu-doped ${ }^{26,36}$ or Sb-doped $\mathrm{SnO}_{2}{ }^{37}$. However the scope here is not to model it but only to report the effect of monochromatic light excitation and current decay, and besides, to show the much faster effect of carrier capture compared to $\mathrm{SnO}_{2}$. Then, the persistent photoconductivity
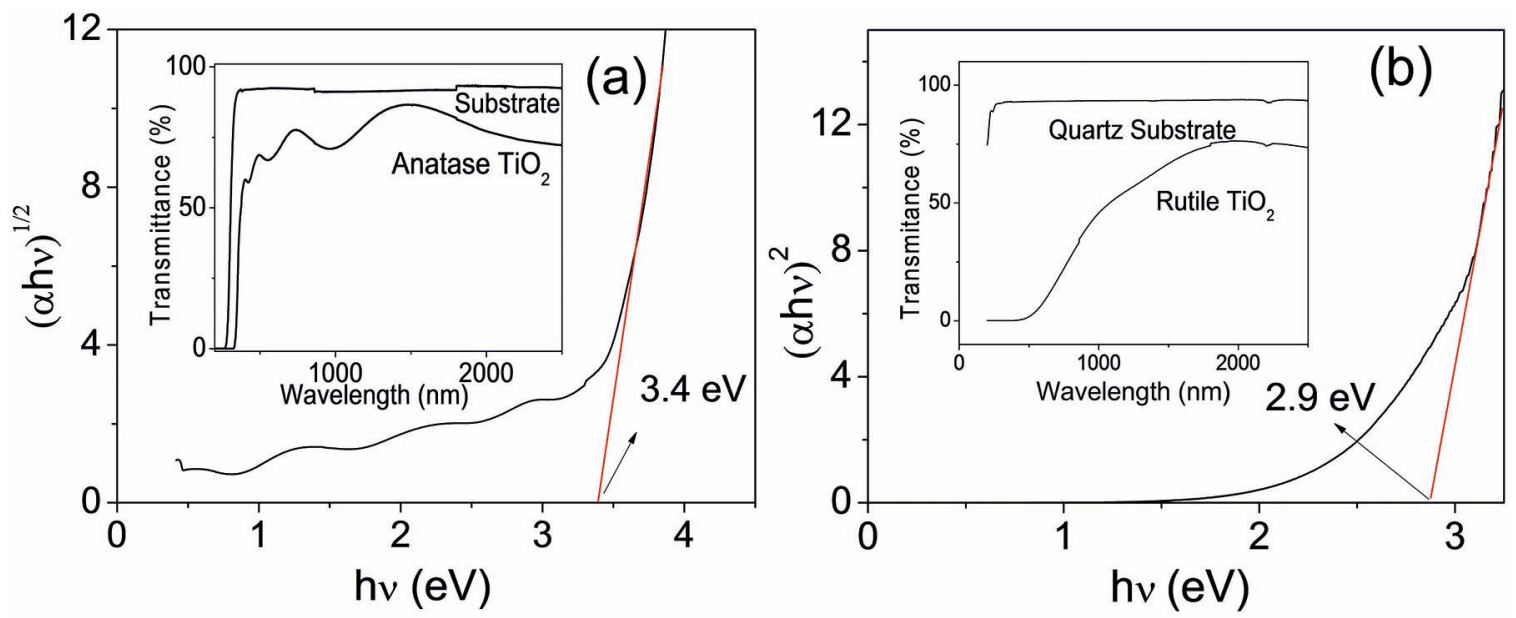

Figure 5. (a) Evaluation of bandgap for anatase structure of $\mathrm{TiO}_{2}$ thin film, obtained from absorbance data. Anatase has indirect transition. Inset: Transmittance spectra for $\mathrm{TiO}_{2}$ thin film deposited on soda-lime glass substrate. (b) Evaluation of bandgap for rutile structure of $\mathrm{TiO}_{2}$ thin film. Rutile has direct transition. Inset: Transmittance for $\mathrm{TiO}_{2}$ thin film deposited on quartz substrate. 


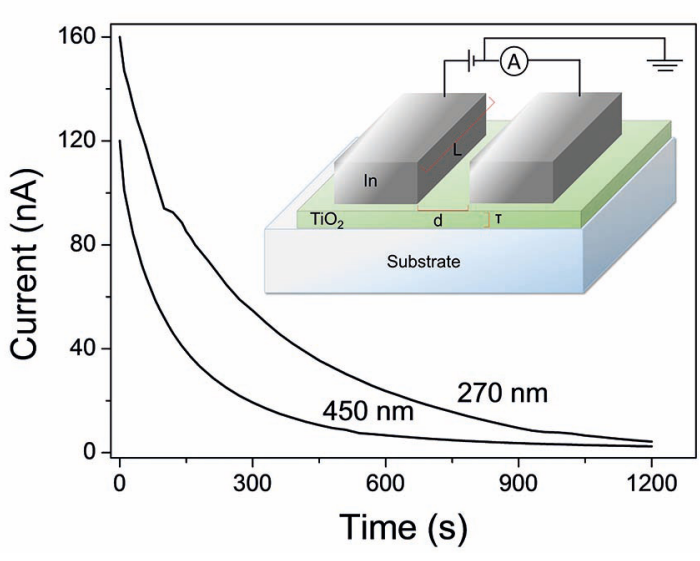

Figure 6. Decay of photoinduced current in $\mathrm{TiO}_{2}$ thin films, with fixed potential of $20 \mathrm{~V}$ and excitation with distinct light sources for 5 minutes. LED source: $15 \mathrm{~W}$, deuterium source: $30 \mathrm{~W}$ (previous to the interference filter). A very quick response was obtained, which sharply increases sample conductivity at the end of the excitation. Inset shows sample layout, with electrical connections and dimensions: film thickness $(\tau)$, distance (d) and width (L) of the contacts.

(PPC) phenomena observed at low temperature for $\mathrm{SnO}_{2}$ (mainly at $70 \mathrm{~K}$ ), is not expected for $\mathrm{TiO}_{2}$. However, the decay can be modeled, because the sample conductance is a function of time, for a fixed temperature, being given by:

$$
G(t)=\left[K_{s} \cdot n(t) \cdot \mu(t) \cdot q\right]
$$

where $K_{s}$ is a constant, $n(t)$ is the time dependent free carrier concentration, $\mu(t)$ is the time dependent electronic mobility, and $q$ is the electron charge.

The observed decay of conductivity as function of time means that the resistance of the film increases with time. The decay of photo-induced electrons (n) from the conduction band to the trapping defect is given by a simple differential equation ${ }^{38}$, whose solution was previously published ${ }^{36,37}$. Considering that in this sort of oxides, the mobility $(\mu)$ is dominated by the grain boundary scattering, we may neglect bulk scattering mechanisms (phonon and ionized impurity). Then, electrical transport, dominated by grain boundary scattering, is an adequate hypothesis, since the grain size is very small. The mobility due to grain boundary scattering is proportional to $\mathrm{T}^{-1 / 2} \exp \left(-\varphi \mathrm{k}^{-1} \mathrm{~T}^{-1}\right)^{39}$, where $\varphi$ is the grain boundary potential barrier. The evaluation of current decay for a complete set of temperatures lead to ${ }^{36}$ :

$\dot{R}=\operatorname{Tan}=K_{D} \cdot T \cdot \exp \left[-\left(E_{\text {cap }}-\phi\right) \cdot(k T)^{-1}\right]$

where $\dot{R}_{R}$ is the tangent of the time-dependent resistance, $\mathrm{K}_{\mathrm{D}}$ is a constant and $E_{c a p}$ is the capture energy for trapping by the dominant defect. Therefore a plot of $\ln \left(\operatorname{Tan} . T^{I}\right)$ as function of $\mathrm{T}^{-1}$ yields the quantity $\left(E_{c a p}-\varphi\right)$ directly from the curve inclination. Figure 6 allows identifying that each curve has distinct rates, which means that its slope will provide distinct values for $\left(E_{c a p}-\varphi\right)$, then belonging to capture due to distinct energy levels (defects). For the results reported in this paper, it is enough to relate that although capture belonging to distinct energy levels takes place, the excitation of this sample can be similar, independent of excitation energy, due to the presence of distinct phases in the material, which have distinct bandgap energies. A modeling to a roomtemperature approach has been recently used for $\mathrm{GaAs} / \mathrm{SnO}_{2}$ heterojunctions ${ }^{40}$, where it was proposed that the current decay comes from a combination of two factors: a variation in the concentration of scattering centers as the electron capture takes place as well as distinct trapping centers at different time intervals, such as doping centers and oxygen vacancies, which are also widely present in the $\mathrm{TiO}_{2}$ samples.

\section{Conclusion}

Although thermal annealing is responsible for phase transition anatase/rutile in $\mathrm{TiO}_{2}$, the thermal annealing procedures used in this work showed incomplete phase transformation, even with applying pressure for pellet sample conformation. Characterization of optical, structural and electrical properties by photoluminescence, X-ray diffraction and transient decay of photoinduced current reveal that the presence of a combination of these two phases may be responsible for the observed properties of the sample. In general, a thermal annealing at $500^{\circ} \mathrm{C}$ leads to a sample with dominant anatase phase properties whereas a thermal annealing at $1000^{\circ} \mathrm{C}$ lead to a rutile dominant phase properties.

Irradiation with monochromatic light strongly affects the thin film conductivity, but the energy range (above or below the bandgap energy) does not seem to affect the excitation behavior, which is associated either with the ionization of intrabandgap states or to crystallites belonging to phases with distinct bandgaps.

The observed properties of this material indicates a high sensitivity for excitation with monochromatic light, which can be tuned, in order to choose the convenient phase properties, Besides, a fast decay to the ground state indicates a suitable property for applications in opto-electronic speedy devices.

\section{Acknowledgements}

We thank the financial support of Capes and FAPESP. We also thank Prof. P. L. Noronha and L. D. Trino for help in the synthesis of $\mathrm{TiO}_{2}$ solution. We also thank Prof. J. H. D. da Silva and N. F. Azevedo for help in transmittance measurement in the UV -Vis region, and Prof D. I. dos Santos for help in measurements of TG / DTA and XRD, which were performed at Multiuser Lab. at UNESP/DF Campus Bauru.

\section{References}

1. Park YR, Kim KJ. Structural and optical properties of rutile and anatase $\mathrm{TiO}_{2}$ thin films: Effects of Co doping. Thin Solid Films. 2005;84(1-2):34-38. DOI: 10.1016/j.tsf.2005.01.039. 
2. Hanaor DAH, Sorrel CC. Review of the anatase to rutile phase transformation. Journal of Materials Science. 2011;46(4):855874. DOI: $10.1007 / \mathrm{s} 10853-010-5113-0$.

3. Mikami M, Nakamura S, Kitao O, Arakawa H, Gonze X. FirstPrinciples Study of Titanium Dioxide: Rutile and Anatase. Japanese Journal of Applied Physics. 2000;39(Pt 2 8B):L847. DOI: $10.1143 /$ JJAP.39.L847.

4. Carp O, Huisman CL, Reller A. Photoinduced reactivity of titanium dioxide. Progress in Solid State Chemistry. 2004;32(1-2):33177. DOI: $10.1016 /$ j.progsolidstchem.2004.08.001.

5. Kalyanasundaram K, ed. Dye-Sensitized Solar Cells. Lausanne: EPFL Press; 2010.

6. Fujishima A. Electrochemical Photolysis of Water at a Semiconductor Electrode. Nature. 1972;238:37-38. DOI: 10.1038/238037a0.

7. Zeng $\mathrm{W}$, Liu $\mathrm{T}$, Wang $\mathrm{Z}$. Impact of $\mathrm{Nb}$ doping on gas-sensing performance of $\mathrm{TiO}_{2}$ thick-film sensors. Sensors and Actuators B: Chemical. 2012;166-167:141-149. DOI: 10.1016/j. snb.2012.02.016.

8. Garzella C, Comini E, Tempesti E, Frigeri C, Sberveglieri G. $\mathrm{TiO}_{2}$ thin films by a novel sol-gel processing for gas sensor applications. Sensors and Actuators B: Chemical. 2000;68(13):189-196. DOI: 10.1016/S0925-4005(00)00428-7.

9. Ding K, Miao Z, Hu B, An G, Sun Z, Han B, et al. Study on the Anatase to Rutile Phase Transformation and Controlled Synthesis of Rutile Nanocrystals with the Assistance of Ionic Liquid. Langmuir. 2010;26(12):10294-10302. DOI: 10.1021/ la100468e.

10. Choudhury B, Choudhury A. Local structure modification and phase transformation of $\mathrm{TiO}_{2}$ nanoparticles initiated by oxygen defects, grain size, and annealing temperature. International Nano Letters. 2013;3(1):55. DOI: 10.1186/2228-5326-3-55.

11. Kandiel TA, Robben L, Alkaim A, Bahnemann D. Brookite versus anatase $\mathrm{TiO}_{2}$ photocatalysts: phase transformations and photocatalytic activities. Photochemical \& Photobiological Sciences, 2013;12(4):602-609. DOI: 10.1039/C2PP25217A.

12. Su C, Hong BY, Tseng CM. Sol-gel preparation and photocatalysis of titanium dioxide. Catalysis Today. 2004;96(3):119-126. DOI: 10.1016/j.cattod.2004.06.132.

13. Sun Y, Egawa T, Zhang L, Yao X. High Anatase-Rutile Transformation Temperature of Anatase Titania Nanoparticles Prepared by Metalorganic Chemical Vapor Deposition. Japanese Journal of Applied Physics. 2002;41(Pt 2 8B):L945. DOI: 10.1143/JJAP.41.1945.

14. Sivakumar S, Pillai PK, Mukundan P, Warrier KGK. Sol-gel synthesis of nanosized anatase from titanyl sulfate. Material Letters. 2002;57(2):330-335. DOI: PII: S0167-577X(02)00786-3.

15. Watson SS, Beydoun D, Scott JA, Amal R. The effect of preparation method on the photoactivity of crystalline titanium dioxide particles. Chemical Engineering Journal. 2003;95(13):213-220. DOI:10.1016/S1385-8947(03)00107-4

16. Guo G, Ito A, Goto T, Tu R, Wang, Shien Q, et al. Preparation of rutile $\mathrm{TiO}_{2}$ thin films by laser chemical vapor deposition method. Journal of Advanced Ceramics. 2013;2(2):162-166. DOI: $10.1007 / \mathrm{s} 40145-013-0056-\mathrm{y}$.
17. Okuya M, Nakade K, Kaneko S. Porous $\mathrm{TiO}_{2}$ thin films synthesized by a spray pyrolysis deposition (SPD) technique and their application to dye-sensitized solar cells. Solar Energy Materials and Solar Cells. 2002;70(4):425-435. DOI: 10.1016/ S0927-0248(01)00033-2.

18. Cho IS, Choi J, Zhang K, Kim SJ, Jeong MJ, Cai L, et al. Highly Efficient Solar Water Splitting from Transferred $\mathrm{TiO}_{2}$ Nanotube Arrays. Nano Letters. 2015;15:5709-5715. DOI: 10.1021/acsnanolett.5b01406.

19. Mor GK, Varghese OK, Paulose M, Grimes CA. Transparent Highly Ordered $\mathrm{TiO}_{2}$ Nanotube Arrays via Anodization of Titanium Thin Films. Advanced Functional Materials. 2005;15(8):12911296. DOI: $10.1002 / \mathrm{adfm} .200500096$

20. Mor GK, Shankar K, Paulose M, Varghese OK, Grimes CA. Use of Highly-Ordered $\mathrm{TiO}_{2}$ Nanotube Arrays in Dye-Sensitized Solar Cells. Nano Letters. 2006;6(2):215-218. DOI: 10.1021/ $\mathrm{n} 1052099 \mathrm{j}$.

21. Tang H, Prasad K, Sanjinès R, Schmid PE, Lévy F. Electrical and optical properties of $\mathrm{TiO}_{2}$ anatase thin films. Journal of Applied Physics. 1993;75(4):2042-2047. DOI: 10.1063/1.356306.

22. Nasr M, Chaaya AA, Abboud N, Bechelany M, Viter R, Eid C, et al. Photoluminescence: A very sensitive tool to detect the presence of anatase in rutile phase electrospun $\mathrm{TiO}_{2}$ nanofibers. Superlattices and Microstructures. 2015;77:18-24. DOI: 10.1016/j.spmi.2014.10.034.

23. Wang X, Feng Z, Shi J, Guoqing J, Shen S, Zhou J, et al. Trap states and carrier dynamics of $\mathrm{TiO}_{2}$ studied by photoluminescence spectroscopy under weak excitation condition. Physical Chemistry Chemical Physics. 2010;12(26):7083-7090. DOI: 10.1039/B925277K.

24. Cullity BD, Stock R. Elements of X-Ray Diffraction. $3^{\text {rd }}$ Ed. New Jersey: Prentice Hall; 2001.

25. Boratto MH, Scalvi LVA, Goncharova LV, Fanchini G. Effects of Solution History on Sol-Gel Processed Tin-Oxide ThinFilm Transistors. Journal of the American Ceramic Society. 2016;92(12):4000-4006. DOI: 10.1111/jace.14459.

26. Ravaro LP, Scalvi LVA, Boratto MH. Improved electrical transport in lightly Er-doped sol-gel spin-coating $\mathrm{SnO}_{2}$ thin films, processed by photolithography. Applied Physics A. 2015;118(4):1419-1427. DOI: 10.1007/s00339-014-8900-7.

27. Bickley RI, Gonzalez-Carreno T, Lees JS, Palmisano, L, Tilley RJD. A structural investigation of titanium dioxide photocatalysts. Journal of Solid State Chemistry. 1991;92(1):178-190. DOI: 10.1016/0022-4596(91)90255-G.

28. Zhang J, Xu Q, Li M, Feng Z, Li C. UV Raman Spectroscopic Study on $\mathrm{TiO}_{2}$. II. Effect of Nanoparticle Size on the Outer/Inner Phase Transformations. The Journal of Physical Chemistry $C$. 2009;113(5):1698-1704. DOI: 10.1021/jp808013k.

29. Madarász J, Okuya M, Varga PP, Kaneko S, Pokol G. TG/ DTA-EGA-MS studies on titania precursors with low content of organics for porous thin films of $\mathrm{TiO}_{2}$. Journal of Analytical and Applied Pyrolysis. 2007;79(1-2):479-483. DOI: 10.1016/j. jaap.2006.12.026. 
30. Satoh N, Nakashima, Yamamoto K. Metastability of anatase: size dependent and irreversible anatase-rutile phase transition in atomic-level precise titania. Scientific Reports. 2013;3:1959. DOI: $10.1038 /$ srep01959.

31. Sonawane RS, Hegde SG, Dongare MK. Preparation of titanium(IV) oxide thin film photocatalyst by sol-gel dip coating. Materials Chemistry and Physics. 2003;77(3):744-750. DOI: 10.1016/ S0254-0584(02)00138-4.

32. Plugaru R, Cremades A, Piqueras J. The effect of annealing in different atmospheres on the luminescence of polycrystalline $\mathrm{TiO}_{2}$. Journal of Physics: Condensed Matter. 2004;16(2):S261-S268. DOI: $10.1088 / 0953-8984 / 16 / 2 / 031$.

33. Nemashkalo AB, Busko TO, Peters RM, Dmytrenko OP, Kulish MP, Vityuk NV, et al. Electronic band structure studies of anatase $\mathrm{TiO}_{2}$ thin films modified with $\mathrm{Ag}, \mathrm{Au}$, or $\mathrm{ZrO}_{2}$ nanophases. Physica Status Solidi B. 2016;253(9):1754-1764. DOI: 10.1002/ pssb.201600042.

34. Tang H, Berger H, Schmid PE, Levy F. Optical Properties of Anatase $\left(\mathrm{TiO}_{2}\right.$ ). Solid State Communications. 1994;92(3):267-271.
35. Cardoso WS, Longo C, De Paoli MA. Preparação de eletrodos opticamente transparentes. Química Nova. 2005;28(2):345-349. DOI: $10.1590 / \mathrm{S} 0100-40422005000200030$.

36. Morais EA, Scalvi LVA, Cavalheiro AA, Tabata A, Oliveira JBB. Rare earth centers properties and electron trapping in $\mathrm{SnO}_{2}$ thin films produced by sol-gel route. Journal of Non-Crystalline Solids. 2008;354(42-44):4840-4845. DOI: 10.1016/j.jnoncrysol.2008.04.029.

37. Floriano EA, Scalvi LVA, Sambrano JR, de Andrade A. Decay of photo-induced conductivity in Sb-doped $\mathrm{SnO}_{2}$ thin films, using monochromatic light of about bandgap energy. Applied Surface Science. 2013;267:164-168. DOI: 10.1016/j.apsusc.2012.09.003.

38. Dobson TW, Scalvi LVA, Wager JF. Transient decay of persistent photoconductivity in $\mathrm{Al}_{0.3} \mathrm{Ga}_{0.7}$ As. Journal of Applied Physics. 1990;68(2):601-605. DOI: 10.1063/1.346785.

39. Zhang DH, Ma HL. Scattering mechanisms of charge carriers in transparent conducting oxide films. Applied Physics A. 1996;62(5):487-492. DOI: 10.1007/BF01567122.

40. Bueno CF, Scalvi LVA. On the electrical properties of distinct $\mathrm{Eu}^{3+}$ emission centers in the heterojunction $\mathrm{GaAs} / \mathrm{SnO}_{2}$. Thin Solid Films. 2016;612:303-309. DOI: 10.1016/j.tsf.2016.06.008. 